\title{
EINE BEMERKUNG ÜBER QUASIELLIPTISCHE LINEARE DIFFERENTIALOPERATOREN
}

\author{
VEIKKO T. PURMONEN
}

Neulich haben V. G. Maz'ja und I. V. Gel'man [5] als eine Folgerung ihrer übrigen Resultate notwendige und hinreichende Bedingungen für die Abschätzung

$$
\|R(D) u\|^{2} \leqq C\left(\|P(D) u\|^{2}+\sum\left|\gamma_{0} Q_{j}(D) u\right|_{\mu-\mu_{j}-q_{n} / 2}^{2}\right), \quad u \in C_{0}^{\infty}\left[\overline{\boldsymbol{R}}_{+}^{n}\right],
$$

gewonnen, wobei $P(D)$ einen quasielliptischen Operator und $R(D)$ einen Operator, der nicht stärker als $P(D)$ ist, sowie $Q_{j}(D)$ Randoperatoren bezeichnen.

Das Ziel dieser Note ist, einen direkten Beweis für dieses Ergebnis unter Benutzung der Methoden von Schechter [6], [7] geben, mit denen auch Matsuzawa 4] ein ähnliches Resultat für die Hinlänglichkeit in einem Spezialfall erhalten hat.

\section{Problemstellung}

1.1. Für zwei Punkte $y=\left(y_{1}, \ldots, y_{n}\right)$ und $\eta=\left(\eta_{1}, \ldots, \eta_{n}\right)$ des euklidischen Raumes $\boldsymbol{R}^{n}$ setzen wir $\langle y, \eta\rangle=y_{1} \eta_{1}+\ldots+y_{n} \eta_{n}$, und es sei $\boldsymbol{R}_{+}^{n}=\left\{y \in \boldsymbol{R}^{n} \mid y_{n}>0\right\}$, $\overline{\boldsymbol{R}}_{+}^{n}=\left\{y \in \boldsymbol{R}^{n} \mid y_{n} \geqq 0\right\}$. Im weiteren ist zweckmäßig $y=(x, t)=\left(x_{1}, \ldots, x_{n-1}, t\right)$ und $\eta=(\xi, \zeta)=\left(\xi_{1}, \ldots, \xi_{n-1}, \zeta\right)$ zu schreiben. Mit Hilfe der Fouriertransformationen $\mathscr{F}_{x}$ in $\boldsymbol{R}^{n-1}$ und $\mathscr{F}_{t}$ in $\boldsymbol{R}$ werden für eine geeignete Funktion $u$ von $y$ die partiellen Fouriertransformierten durch

bzw.

$$
\left(\mathscr{F}_{x} u\right)(\xi, t)=\pi_{n-1} \int e^{-i\langle x, \xi\rangle} u(x, t) d x
$$

$$
\left(\mathscr{F}_{t} u\right)(x, \zeta)=\pi_{1} \int e^{-i t \zeta} u(x, t) d t
$$

definiert (vgl. [3], S. 24), wobei $\pi_{k}=(2 \pi)^{-k / 2}, k=1,2, \ldots$, ist.

Mit $C_{0}^{\infty}\left[\overline{\boldsymbol{R}}_{+}^{n}\right]$ bezeichnen wir die Menge der Einschränkungen von $C_{0}^{\infty}\left(\boldsymbol{R}^{n}\right)$ Funktionen auf $\overline{\boldsymbol{R}}_{+}^{n}$. Die Restriktion $\gamma_{0} u$ von $u \in C_{0}^{\infty}\left[\overline{\boldsymbol{R}}_{+}^{n}\right]$ auf $\boldsymbol{R}^{n-1}$ wird durch $\left(\gamma_{0} u\right)(x)=u(x, 0)$ erklärt.

1.2. Es seien ganze Zahlen $m_{k} \geqq 1, \quad k=1, \ldots, n$, festgelegt, und sei $\mu=\max \left\{m_{k} \mid 1 \leqq k \leqq n\right\} \quad$ sowie $\quad q_{k}=\mu / m_{k}, \quad k=1, \ldots, n, \quad$ und $\quad q=\left(q^{\prime}, q_{n}\right)=$ $=\left(q_{1}, \ldots, q_{n-1}, q_{n}\right)$. 
Eine in $\boldsymbol{R}^{n} \backslash\{0\}$ definierte Funktion $h: \eta=(\xi, \zeta) \mapsto h(\xi, \zeta)$ nennt man $q$-homogen vom Grad $s \in \boldsymbol{R}$ und schreibt $\operatorname{deg}_{q} h=s$, falls für alle $t>0$

gilt, wobei

$$
h\left(t^{q} \eta\right)=h\left(t^{q^{\prime}} \xi, t^{q_{n}} \zeta\right)=t^{s} h(\xi, \zeta)=t^{s} h(\eta)
$$

$$
t^{q} \eta=\left(t^{q^{\prime}} \xi, t^{q_{n}} \zeta\right)=\left(t^{q_{1}} \xi_{1}, \ldots, t^{q_{n-1}} \xi_{n-1}, t^{q_{n}} \zeta\right)
$$

geschrieben ist; analog definiert man für eine Funktion $g: \xi \mapsto g(\xi)$ die $q^{\prime}$-Homogenität vom Grad $s \in \boldsymbol{R}, \operatorname{deg}_{q^{\prime}} g=s$.

Ferner setzen wir

$$
\langle\xi\rangle=\left(\sum_{k=1}^{n-1}\left|\xi_{k}\right|^{m_{k}}\right)^{1 / \mu}, \quad \xi \in \boldsymbol{R}^{n-1},
$$

und erklären in $C_{0}^{\infty}\left(\boldsymbol{R}^{n-1}\right)$ ein Funktional $|\cdot|_{s}, s \geqq 0$, durch

$$
|u|_{s}^{2}=\int\langle\xi\rangle^{2 s}\left|\left(\mathscr{F}_{x} u\right)(\xi)\right|^{2} d \xi, \quad u \in C_{0}^{\infty}\left(\boldsymbol{R}^{n-1}\right) .
$$

Die Norm des Raumes $L^{2}\left(\boldsymbol{R}_{+}^{n}\right)$ wird mit $\|\cdot\|$ bezeichnet.

1.3. Wir betrachten einen partiellen Differentialoperator

$$
P(D)=P\left(D_{x}, D_{t}\right)=\sum a_{p} D^{p}=\sum a_{p} D_{x}^{p^{\prime}} D_{t}^{p_{n}}
$$

mit konstanten Koeffizienten $a_{p} \in \boldsymbol{C}$; hierbei ist $p$ ein Multiindex $p=\left(p^{\prime}, p_{n}\right)=$ $=\left(p_{1}, \ldots, p_{n-1}, p_{n}\right) \in N^{n} \quad$ und $\quad D^{p}=D_{x}^{p^{\prime}} D_{t}^{p_{n}}=D_{1}^{p_{1}} \ldots D_{n-1}^{p_{n}} D_{n}^{p_{n}}$ mit $D=\left(D_{x}, D_{t}\right)=$ $=\left(D_{1}, \ldots, D_{n-1}, D_{n}\right), D_{k}=-i \partial / \partial y_{k}$. Das entsprechende Polynom hat die Gestalt

$$
P(\eta)=P(\xi, \zeta)=\sum a_{p} \eta^{p}=\sum a_{p} \xi^{p^{\prime}} \zeta^{p_{n}},
$$

wobei $\eta^{p}=\xi^{p^{\prime}} \zeta^{p_{n}}=\xi_{1}^{p_{1}} \ldots \xi_{n-1}^{p_{n}} \zeta^{p_{n}}$ ist; die Ordnung des Operators $P(D)$ stimmt also mit dem Grad ord $P(\eta)$ von $P(\eta)$ überein.

Wir setzen voraus:

a) $P(\xi, \zeta)$ ist ein $q$-homogenes Polynom mit $\operatorname{deg}_{q} P=\mu$.

b) $P(\xi, \zeta)$ ist quasielliptisch vom bestimmten Typ $K^{+} \geqq 1$ :

(i) es gilt $P(\xi, \zeta) \neq 0$ für $(\xi, \zeta) \in \boldsymbol{R}^{n} \backslash\{0\}$ oder äquivalenterweise (vgl. [3], S. 103)

$$
|P(\xi, \zeta)| \geqq C_{0}\left(\langle\zeta\rangle^{\mu}+|\zeta|^{m_{n}}\right), \quad(\zeta, \zeta) \in \boldsymbol{R}^{n},
$$

mit einer Konstanten $C_{0}>0$;

(ii) für alle $\xi \in \boldsymbol{R}^{n-1} \backslash\{0\}$ besitzt die Gleichung $P(\xi, z)=0$ genau $K^{+}$Lösungen $z=\zeta(\xi)$ mit positivem Imaginärteil $\operatorname{Im} \zeta(\xi)>0$.

Für $\xi \in \boldsymbol{R}^{n-1} \backslash\{0\}$ seien die verschiedenen Nullstellen des Polynoms $P(\xi, z)$ mit $\zeta_{\alpha}(\xi)$ und die zugehörigen Ordnungen mit $k_{\alpha}(\xi)$ bezeichnet. Dann stellen wir Bedingung (A). Für $\alpha \neq \beta$ gilt

$$
\zeta_{\alpha}(\xi) \neq \zeta_{\beta}(\xi), \quad \xi \in \boldsymbol{R}^{n-1} \backslash\{0\},
$$

d. h. die Ordnung $k_{\alpha}(\xi)$ hängt nicht von $\xi a b$. 
Man kann nun annehmen, daß mit gewissen Zahlen $1 \leqq \lambda^{+} \leqq \lambda$ für die Indexmengen

$$
\Lambda=\{1, \ldots, \lambda\}, \quad \Lambda^{+}=\left\{1, \ldots, \lambda^{+}\right\}, \quad \Lambda^{-}=\Lambda \backslash \Lambda^{+}
$$

die Darstellung

$$
P(\xi, \zeta)=\prod_{\alpha \in \Lambda}\left(\zeta-\zeta_{\alpha}(\xi)\right)^{k_{\alpha}}
$$

besteht (dies bedeutet keine wesentliche Beschränkung, vgl. [2], S. 239) und die Beziehungen

und

gelten. Für das durch

$$
\operatorname{Im} \zeta_{\alpha}(\xi)>0 \quad \text { für } \alpha \in \Lambda^{+}
$$

$$
\operatorname{Im} \zeta_{\alpha}(\xi)<0 \quad \text { für } \alpha \in \Lambda^{-}
$$

$$
P_{+}(\xi, \zeta)=\prod_{\alpha \in \Lambda^{+}}\left(\zeta-\zeta_{\alpha}(\xi)\right)^{k_{\alpha}}
$$

erklärte Polynom $P_{+}(\xi, \zeta)$ ist dann die $\zeta$-Ordnung $\operatorname{ord}_{\zeta} P_{+}(\xi, \zeta)=k_{1}+\ldots+$ $+k_{\lambda^{+}}=K^{+}$.

Es sei jetzt $R(\xi, \zeta)$ ein $q$-homogenes Polynom mit $\operatorname{deg}_{q} R=\mu$ und $M(\xi, \zeta)$ (mit 1 als Hauptkoeffizient von $\zeta$ ) der größte gemeinsame Teiler der Polynome $P_{+}(\xi, \zeta)$ und $R(\xi, \zeta)$. Wir schreiben

und stellen

$$
P_{+}^{\prime}(\xi, \zeta)=P_{+}(\xi, \zeta) / M(\xi, \zeta)
$$

Bedingung (B). Der Grad von $P_{+}^{\prime}(\xi, \zeta)$ in $\zeta$ ist positiv und bleibt konstant für $\xi \in \boldsymbol{R}^{n-1} \backslash\{0\}$.

Demzufolge können wir voraussetzen, daß eine Zahl $\lambda^{\prime}, 1 \leqq \lambda^{\prime} \leqq \lambda^{+}$, so existiert, daß mit $A^{\prime}=\left\{1, \ldots, \lambda^{\prime}\right\}$

$$
P_{+}^{\prime}(\xi, \zeta)=\prod_{\alpha \in \Lambda^{\prime}}\left(\zeta-\zeta_{\alpha}(\xi)\right)^{x_{\alpha}}
$$

gilt, wobei $x_{\alpha} \leqq k_{\alpha}$ und $x_{1}+\ldots+x_{\lambda^{\prime}}=x=\operatorname{ord}_{\zeta} P_{+}^{\prime}(\xi, \zeta) \geqq 1$ ist.

Noch sei $x_{\alpha}=k_{\alpha}$ für $\alpha \in \Lambda \backslash \Lambda^{\prime}$ und $\varrho_{\alpha}=\left\{0, \ldots, x_{\alpha}-1\right\}$ für $\alpha \in \Lambda$ gesetzt.

1.4. Das Ergebnis von V. G. Maz'ja und I. V. Gel'man, dem wir hier einen direkten Beweis geben wollen, lautet nun wie folgt (vgl. [5], S. 254):

Satz 1.1. Es seien $P(\xi, \zeta)$ ein q-homogenes quasielliptisches Polynom vom bestimmten Typ und $R(\xi, \zeta)$ ein q-homogenes Polynom mit $\operatorname{deg}_{q} P=\operatorname{deg}_{q} R=\mu$ derart, daß die Bedingungen $(A)$ und $(B)$ erfüllt sind, $\operatorname{ord}_{\zeta} P_{+}^{\prime}(\xi, \zeta)=\chi$. Ferner seien $Q_{j}(\xi, \zeta), j=1, \ldots, x, q$-homogene Polynome mit $\operatorname{deg}_{q} Q_{j}=\mu_{j} \leqq \mu-q_{n}$.

Die Abschätzung

$$
\|R(D) u\|^{2} \leqq C\left(\|P(D) u\|^{2}+\sum_{j=1}^{\varkappa}\left|\gamma_{0} Q_{j}(D) u\right|_{\mu-\mu_{j}-q_{n} / 2}^{2}\right), \quad u \in C_{0}^{\infty}\left[\bar{R}_{+}^{n}\right]
$$


gilt dann und nur dann, wenn die folgenden Bedingungen (I) und (II) für alle $\xi \in \boldsymbol{R}^{n-1} \backslash\{0\}$ erfüllt sind:

(I) Für jedes $j, 1 \leqq j \leqq \varkappa$, ist $Q_{j}(\xi, \zeta) \equiv 0 \bmod M(\xi, \zeta)$.

(II) Die Polynome $Q_{j}(\xi, \zeta), j=1, \ldots, x$, sind linear unabhängig modulo $P_{+}(\xi, \zeta)$.

\section{Die Hinlänglichkeit der Bedingungen}

2.1. Die Bedingungen (I) und (II) sind hinreichend, falls mit den Bezeichnungen

$$
\begin{aligned}
& P^{\prime}(\xi, \zeta)=P(\xi, \zeta) / M(\xi, \zeta), \\
& R^{\prime}(\xi, \zeta)=R(\xi, \zeta) / M(\xi, \zeta), \\
& Q_{j}^{\prime}(\xi, \zeta)=Q_{j}(\xi, \zeta) / M(\xi, \zeta), \quad j=1, \ldots, \varkappa,
\end{aligned}
$$

gilt:

Satz 2.1. Unter den Voraussetzungen von Satz 1.1 besteht die Abschätzung

$$
\left\|R^{\prime}(D) v\right\|^{2} \leqq C\left(\left\|P^{\prime}(D) v\right\|^{2}+\sum_{j=1}^{x}\left|\gamma_{0} Q_{j}^{\prime}(D) v\right|_{\mu-\mu_{j}-q_{n} / 2}^{2}\right), \quad v \in C_{0}^{\infty}\left[\overline{\boldsymbol{R}}_{+}^{n}\right],
$$

falls die folgende Bedingung (III) für alle $\xi \in \boldsymbol{R}^{n-1} \backslash\{0\}$ erfüllt ist:

(III) Die Polynome $Q_{j}^{\prime}(\xi, \zeta), j=1, \ldots, x$, sind linear unabhängig modulo $P_{+}^{\prime}(\xi, \zeta)$.

In der Tat, aus den Bedingungen (I) und (II) folgt Bedingung (III) und für $u \in C_{0}^{\infty}\left[\overline{\boldsymbol{R}}_{+}^{n}\right]$ ist $v=M(D) u \in C_{0}^{\infty}\left[\overline{\boldsymbol{R}}_{+}^{n}\right]$, so daß Abschätzung (1.1) sich aus (2.1) ergibt.

2.2. Es sei $\xi \in \boldsymbol{R}^{n-1} \backslash\{0\}$. Wir setzen

$$
\begin{array}{ll}
P_{\alpha}^{\prime}(\xi, \zeta)=\left(\zeta-\zeta_{\alpha}(\xi)\right)^{-x_{\alpha}} P^{\prime}(\xi, \zeta) & \text { für } \alpha \in \Lambda^{\prime} \cup \Lambda^{-}, \\
P_{+\alpha}^{\prime}(\xi, \zeta)=\left(\zeta-\zeta_{\alpha}(\xi)\right)^{-x_{\alpha}} P_{+}^{\prime}(\xi, \zeta) & \text { für } \alpha \in \Lambda^{\prime}, \\
P_{-\alpha}(\zeta, \zeta)=\left(\zeta-\zeta_{\alpha}(\xi)\right)^{-x_{\alpha}} P_{-}(\xi, \zeta) & \text { für } \alpha \in \Lambda^{-} .
\end{array}
$$

Dann gibt es ein solches $r_{0}(\xi)$ und solche Polynome (in $\left.\zeta\right) R_{+}(\xi, \zeta)$ und $R_{-}(\xi, \zeta)$, daß die Formel

und folglich

$$
\frac{R(\xi, \zeta)}{P(\xi, \zeta)}=r_{0}(\xi)+\frac{R_{+}(\xi, \zeta)}{P_{+}(\xi, \zeta)}+\frac{R_{-}(\xi, \zeta)}{P_{-}(\xi, \zeta)}
$$

gilt, wobei

$$
\frac{R^{\prime}(\xi, \zeta)}{P^{\prime}(\xi, \zeta)}=r_{0}(\xi)+\frac{R_{+}^{\prime}(\xi, \zeta)}{P_{+}^{\prime}(\xi, \zeta)}+\frac{R_{-}(\xi, \zeta)}{P_{-}(\xi, \zeta)}
$$

und

$$
R^{\prime}(\xi, \zeta)=R(\xi, \zeta) / M(\xi, \zeta)
$$

$$
R_{+}^{\prime}(\xi, \zeta)=R_{+}(\xi, \zeta) / M(\xi, \zeta)
$$


geschrieben wurden. Mit Hilfe der verallgemeinerten Lagrangeschen Interpolationsformel (Lagrange-Sylvester-Formel) erhält man jetzt

mit

$$
\begin{aligned}
\frac{R^{\prime}(\xi, \zeta)}{P^{\prime}(\xi, \zeta)}= & r_{0}(\xi)+\sum_{\alpha \in \Lambda^{\prime}} \sum_{\beta \in \varrho_{\alpha}} r_{\alpha \beta}^{+}(\xi)\left(\zeta-\zeta_{\alpha}(\xi)\right)^{\beta-x_{\alpha}} \\
& +\sum_{\alpha \in \Lambda^{-}} \sum_{\beta \in \varrho_{\alpha}} r_{\alpha \beta}^{-}(\xi)\left(\zeta-\zeta_{\alpha}(\xi)\right)^{\beta-x_{\alpha}}
\end{aligned}
$$

und

Somit gilt

$$
r_{\alpha \beta}^{+}(\xi)=\left.\frac{1}{\beta !}\left(\frac{\partial^{\beta}}{\partial \zeta^{\beta}} \frac{R_{+}^{\prime}(\xi, \zeta)}{P_{+\alpha}^{\prime}(\xi, \zeta)}\right)\right|_{\zeta=\zeta_{\alpha}(\xi)}, \quad \alpha \in \Lambda^{\prime}, \quad \beta \in \varrho_{\alpha}
$$

$$
r_{\alpha \beta}^{-}(\xi)=\left.\frac{1}{\beta !}\left(\frac{\partial^{\beta}}{\partial \zeta^{\beta}} \frac{R_{-}(\xi, \zeta)}{P_{-\alpha}(\xi, \zeta)}\right)\right|_{\zeta=\zeta_{\alpha}(\xi)}, \quad \alpha \in \Lambda^{-}, \beta \in \varrho_{\alpha}
$$

Hiermit ist für $\varphi \in C_{0}^{\infty}\left[\overline{\boldsymbol{R}}_{+}\right]$

$$
\begin{aligned}
R^{\prime}(\xi, \zeta)= & r_{0}(\xi) P^{\prime}(\xi, \zeta) \\
& +\sum_{\alpha \in \Lambda^{\prime}} \sum_{\beta \in Q_{\alpha}} r_{\alpha \beta}^{+}(\xi)\left(\zeta-\zeta_{\alpha}(\xi)\right)^{\beta} P_{\alpha}^{\prime}(\xi, \zeta) \\
& +\sum_{\alpha \in \Lambda^{-}} \sum_{\beta \in \boldsymbol{Q}_{\alpha}} r_{\alpha \beta}^{-}(\xi)\left(\zeta-\zeta_{\alpha}(\xi)\right)^{\beta} P_{\alpha}^{\prime}(\xi, \zeta) .
\end{aligned}
$$

2.3. Zunächst wird der Fall $n=1$ besonders untersucht. Formel (2.4) hat dann die Gestalt

$$
\begin{aligned}
R^{\prime}(\zeta) & =r_{0} P^{\prime}(\zeta)+\sum_{\alpha \in \Lambda^{\prime}} \sum_{\beta \in \varrho_{\alpha}} r_{\alpha \beta}^{+}\left(\zeta-\zeta_{\alpha}\right)^{\beta} P_{\alpha}^{\prime}(\zeta) \\
& +\sum_{\alpha \in \Lambda^{-}} \sum_{\beta \in \varrho_{\alpha}} r_{\alpha \beta}^{-}\left(\zeta-\zeta_{\alpha}\right)^{\beta} P_{\alpha}^{\prime}(\zeta) .
\end{aligned}
$$

$$
\begin{aligned}
R^{\prime}\left(D_{t}\right) \tilde{\varphi}= & r_{0} P^{\prime}\left(D_{t}\right) \tilde{\varphi} \\
& +\sum_{\alpha \in \Lambda^{\prime}} \sum_{\beta \in \varrho_{\alpha}} r_{\alpha \beta}^{+}\left(D_{t}-\zeta_{\alpha}\right)^{\beta} P_{\alpha}^{\prime}\left(D_{t}\right) \tilde{\varphi} \\
& +\sum_{\alpha \in \Lambda^{-}} \sum_{\beta \in \varrho_{\alpha}} r_{\alpha \beta}^{-}\left(D_{t}-\zeta_{\alpha}\right)^{\beta} P_{\alpha}^{\prime}\left(D_{t}\right) \tilde{\varphi},
\end{aligned}
$$

wobei $\tilde{\varphi}$ die Nullfortsetzung von $\varphi$ für $t<0$ bezeichnet.

Setzt man

$$
f(t)=P^{\prime}\left(D_{t}\right) \tilde{\varphi}(t)= \begin{cases}P^{\prime}\left(D_{t}\right) \varphi(t) & \text { für } t \geqq 0 \\ 0 & \text { für } t<0,\end{cases}
$$

so gilt (man siehe 4.1)

Lemma 2.2. Wenn $\varphi \in C_{0}^{\infty}\left[\overline{\boldsymbol{R}}_{+}\right]$und $f$ durch (2.7) erklärt ist, dann besteht für alle $\alpha \in \Lambda^{\prime} \cup \Lambda^{-}, \beta \in \varrho_{\alpha}$ die Gleichung

$$
\mathscr{F}_{t}\left(\left(D_{t}-\zeta_{\alpha}\right)^{\beta} P_{\alpha}^{\prime}\left(D_{t}\right) \tilde{\varphi}\right)(\zeta)=\left(\zeta-\zeta_{\alpha}\right)^{\beta-x_{\alpha}}\left(\mathscr{F}_{t} f\right)(\zeta)-i \pi_{1} \sum_{k=1}^{x_{\alpha}-\beta}\left(\zeta-\zeta_{\alpha}\right)^{k-x_{\alpha}+\beta-1} W_{\alpha k}
$$

mit

$$
W_{\alpha k}=\gamma_{0}\left(D_{t}-\zeta_{\alpha}\right)^{\alpha_{\alpha}-k} P_{\alpha}^{\prime}\left(D_{t}\right) \varphi(t)
$$


Mit Hilfe von Lemma 2.2 erhalten wir aus (2.6)

$$
\begin{aligned}
\mathscr{F}_{t}\left(R^{\prime}\left(D_{t}\right) \tilde{\varphi}\right)(\zeta)= & r_{0}\left(\mathscr{F}_{t} f\right)(\zeta) \\
& +\sum_{\alpha \in \Lambda^{\prime}} \sum_{\beta \in \ell_{\alpha}} r_{\alpha \beta}^{+}\left(\zeta-\zeta_{\alpha}\right)^{\beta-x_{\alpha}}\left(\mathscr{F}_{t} f\right)(\zeta) \\
& +\sum_{\alpha \in \Lambda^{-}} \sum_{\beta \in e_{\alpha}} r_{\alpha \beta}^{-}\left(\zeta-\zeta_{\alpha}\right)^{\beta-x_{\alpha}}\left(\mathscr{F}_{t} f\right)(\zeta) \\
& -i \pi_{1} \sum_{\alpha \in \Lambda^{\prime}} \sum_{\beta \in \ell_{\alpha}} r_{\alpha \beta}^{+} \sum_{k=1}^{\alpha_{\alpha}-\beta}\left(\zeta-\zeta_{\alpha}\right)^{k-x_{\alpha}+\beta-1} W_{\alpha k} \\
& -i \pi_{1} \sum_{\alpha \in A^{-}} \sum_{\beta \in \ell_{\alpha}} r_{\alpha \beta}^{-} \sum_{k=1}^{\alpha_{\alpha}-\beta}\left(\zeta-\zeta_{\alpha}\right)^{k-x_{\alpha}+\beta-1} W_{\alpha k} .
\end{aligned}
$$

Daher gilt wegen (2.5)

$$
\begin{aligned}
\mathscr{F}_{t}\left(R^{\prime}\left(D_{t}\right) \tilde{\varphi}\right)(\zeta)= & \frac{R^{\prime}(\zeta)}{P^{\prime}(\zeta)}\left(\mathscr{F}_{t} f\right)(\zeta) \\
& -i \pi_{1} \sum_{\alpha \in \Lambda^{\prime}} \sum_{\beta \in \ell_{\alpha}} r_{\alpha \beta}^{+} \sum_{k=1}^{x_{\alpha}-\beta}\left(\zeta-\zeta_{\alpha}\right)^{k-x_{\alpha}+\beta-1} W_{\alpha k} \\
& -i \pi_{1} \sum_{\alpha \in A^{-}} \sum_{\beta \in \ell_{\alpha}} r_{\alpha \beta}^{-} \sum_{k=1}^{x_{\alpha}-\beta}\left(\zeta-\zeta_{\alpha}\right)^{k-x_{\alpha}+\beta-1} W_{\alpha k} .
\end{aligned}
$$

2.4. Unter Berücksichtigung der Parsevalschen Gleichung folgt aus (2.8)

$$
\begin{aligned}
\int_{0}^{\infty}\left|R^{\prime}\left(D_{t}\right) \varphi\right|^{2} d t \leqq & C\left(\sup \left|\frac{R^{\prime}(\zeta)}{P^{\prime}(\zeta)}\right|^{2} \int_{-\infty}^{\infty}|f|^{2} d t\right. \\
& +\sum_{\alpha \in \Lambda^{\prime}} \sum_{\beta \in e_{\alpha}} \sum_{k=1}^{x_{\alpha}-\beta}\left|r_{\alpha \beta}^{+}\right|^{2}\left|W_{\alpha k}\right|^{2} \int_{-\infty}^{\infty} \frac{d \zeta}{\left|\zeta-\zeta_{\alpha}\right|^{2\left(x_{\alpha}-\beta-k+1\right)}} \\
& \left.+\sum_{\alpha \in \Lambda^{-}} \sum_{\beta \in Q_{\alpha}} \sum_{k=1}^{\alpha_{\alpha}-\beta}\left|r_{\alpha \beta}^{-}\right|^{2}\left|W_{\alpha k}\right|^{2} \int_{-\infty}^{\infty} \frac{d \zeta}{\left|\zeta-\zeta_{\alpha}\right|^{2\left(\alpha_{\alpha}-\beta-k+1\right)}}\right)
\end{aligned}
$$

Für die Behandlung der rechten Seite von (2.9) benötigen wir zwei Lemmata (man siehe 4.2).

Lemma 2.3. Für jedes $s=1,2, \ldots$ ist

$$
\int_{-\infty}^{\infty} \frac{d \zeta}{\left|\zeta-\zeta_{\alpha}\right|^{2 s}}=2 \pi \frac{(2(s-1)) !}{((s-1) !)^{2}} \frac{1}{\left|\operatorname{Im} \zeta_{\alpha}\right|^{2 s-1}} .
$$

Lemma 2.4. Ist $\alpha \in \Lambda^{-}$, so gilt für jedes $k=1, \ldots, \chi_{\alpha}$

$$
W_{\alpha k}=\pi_{1} \int_{-\infty}^{\infty} \frac{\left(\mathscr{F}_{t} f\right)(\zeta)}{\left(\zeta-\zeta_{\alpha}\right)^{k}} d \zeta
$$


Mit Hilfe der vorigen Lemmata und der Schwarzschen Ungleichung erhält man jetzt in dem zweiten Glied der rechten Seite von (2.9)

$$
\begin{aligned}
\left|W_{\alpha k}\right|^{2} \int_{-\infty}^{\infty} \frac{d \zeta}{\left|\zeta-\zeta_{\alpha}\right|^{2\left(x_{\alpha}-\beta-k+1\right)}} & \leqq C \int_{-\infty}^{\infty}\left|\mathscr{F}_{t} f\right|^{2} d \zeta \int_{-\infty}^{\infty} \frac{d \zeta}{\left|\zeta-\zeta_{\alpha}\right|^{2 k}} \int_{-\infty}^{\infty} \frac{d \zeta}{\left|\zeta-\zeta_{\alpha}\right|^{2\left(x_{\alpha}-\beta-k+1\right)}} \\
& \leqq C \frac{1}{\left|\operatorname{Im} \zeta_{\alpha}\right|^{2\left(x_{\alpha}-\beta\right)}} \int_{-\infty}^{\infty}|f|^{2} d t
\end{aligned}
$$

und folglich gilt

$$
\begin{aligned}
\int_{0}^{\infty}\left|R^{\prime}\left(D_{t}\right) \varphi\right|^{2} d t \leqq & C\left(\sup \left|\frac{R^{\prime}(\zeta)}{P^{\prime}(\zeta)}\right|^{2} \int_{-\infty}^{\infty}|f|^{2} d t\right. \\
& +\sum_{\alpha \in \Lambda^{\prime}} \sum_{\beta \in \boldsymbol{Q}_{\alpha}} \sum_{k=1}^{x_{\alpha}-\beta}\left|r_{\alpha \beta}^{+}\right|^{2}\left|W_{\alpha k}\right|^{2} \frac{1}{\left|\operatorname{Im} \zeta_{\alpha}\right|^{2\left(x_{\alpha}-\beta-k+1 / 2\right)}} \\
& \left.+\sum_{\alpha \in \Lambda^{-}} \sum_{\beta \in \boldsymbol{Q}_{\alpha}}\left|r_{\alpha \beta}^{-}\right|^{2} \frac{1}{\left|\operatorname{Im} \zeta_{\alpha}\right|^{2\left(x_{\alpha}-\beta\right)}} \int_{-\infty}^{\infty}|f|^{2} d t\right)
\end{aligned}
$$

2.5. Wir gehen auf den allgemeinen Fall zurück. Mit festem $\xi \in R^{n-1} \backslash\{0\}$ hat man nach (2.10)

$$
\begin{aligned}
& \int_{0}^{\infty}\left|R^{\prime}\left(\xi, D_{t}\right)\left(\mathscr{F}_{x} v\right)(\xi, t)\right|^{2} d t \\
& \leqq C\left(\sup _{\zeta}\left|\frac{R^{\prime}(\xi, \zeta)}{P^{\prime}(\xi, \zeta)}\right|^{2} \int_{0}^{\infty}\left|P^{\prime}\left(\xi, D_{t}\right)\left(\mathscr{F}_{x} v\right)(\xi, t)\right|^{2} d t\right. \\
&+\sum_{\alpha \in \Lambda^{\prime}} \sum_{\beta \in Q_{\alpha}} \sum_{k=1}^{x_{\alpha}-\beta}\left|r_{\alpha \beta}^{+}(\xi)\right|^{2}\left|W_{\alpha k}(\xi)\right|^{2} \frac{1}{\left|\operatorname{Im} \zeta_{\alpha}(\xi)\right|^{2\left(x_{\alpha}-\beta-k+1 / 2\right)}} \\
&\left.+\sum_{\alpha \in \Lambda^{-}} \sum_{\beta \in Q_{\alpha}}\left|r_{\alpha \beta}^{-}(\xi)\right|^{2} \frac{1}{\left|\operatorname{Im} \zeta_{\alpha}(\xi)\right|^{2\left(x_{\alpha}-\beta\right)}} \int_{0}^{\infty}\left|P^{\prime}\left(\xi, D_{t}\right)\left(\mathscr{F}_{x} v\right)(\xi, t)\right|^{2} d t\right)
\end{aligned}
$$

mit

$$
W_{\alpha k}(\xi)=\gamma_{0}\left(D_{t}-\zeta_{\alpha}(\xi)\right)^{\alpha_{\alpha}-k} P_{\alpha}^{\prime}\left(\xi, D_{t}\right)\left(\mathscr{F}_{x} v\right)(\xi, t) .
$$

Aufgrund der Voraussetzungen gilt hierbei

$$
\sup _{\zeta}\left|\frac{R^{\prime}(\xi, \zeta)}{P^{\prime}(\xi, \zeta)}\right| \leqq C_{1} \quad \text { für alle } \xi \in R^{n-1} \backslash\{0\}
$$

mit einer Konstanten $C_{1}>0$, und

$$
\zeta_{\alpha} \text { ist } q^{\prime} \text {-homogen (in } \xi \text { ) mit } \operatorname{deg}_{q^{\prime}} \zeta_{\alpha}=q_{n}
$$


infolge der Bedingung (A). Wegen der Stetigkeit schließt man aus (2.13), daß

$$
\left|\operatorname{Im} \zeta_{\alpha}(\xi)\right| \geqq C_{2}\langle\xi\rangle^{q_{n}}, \quad \xi \in \boldsymbol{R}^{n-1} \backslash\{0\},
$$

mit einer Konstanten $C_{2}>0$ gilt.

Noch haben wir (man siehe 4.3)

Lemma 2.5. Es existieren Konstanten $C_{3}>0$ und $C_{4}>0$ derart, daß die Ungleichungen

und

$$
\left|r_{\alpha \beta}^{+}(\xi)\right| \leqq C_{3}\langle\xi\rangle^{\left(\varkappa_{\alpha}-\beta\right) q_{n}}
$$

$$
\left|r_{\alpha \beta}^{-} \dot{\prime}(\xi)\right| \leqq C_{4}\langle\xi\rangle^{\left(\alpha_{\alpha}-\beta\right) q_{n}}
$$

für alle $\xi \in \boldsymbol{R}^{n-1} \backslash\{0\}$ bestehen.

Berücksichtigt man (2.12), (2.14) und Lemma 2.5, so erhält man aus (2.11)

$$
\begin{aligned}
\int_{0}^{\infty}\left|R^{\prime}\left(\xi, D_{t}\right)\left(\mathscr{F}_{x} v\right)(\xi, t)\right|^{2} d t & \\
\leqq & C\left(\int_{0}^{\infty}\left|P^{\prime}\left(\xi, D_{t}\right)\left(\mathscr{F}_{x} v\right)(\xi, t)\right|^{2} d t\right. \\
& +\sum_{\alpha \in A^{\prime}} \sum_{\beta \in e_{\alpha}} \mid\langle\xi\rangle^{\left.\left.\left(x_{\alpha}-\beta-1 / 2\right) q_{n} W_{\alpha, \alpha_{\alpha}-\beta}(\xi)\right|^{2}\right) .}
\end{aligned}
$$

2.6. Wir wenden uns nun an die Polynome $Q_{j}(\xi, \zeta), j=1, \ldots, \varkappa$. Es sei $\xi \in \boldsymbol{R}^{n-1} \backslash\{0\}$. Dann existieren Polynome (in $\zeta$ ) $Q_{j+}(\xi, \zeta)$ und $Q_{j-}(\xi, \zeta)$ mit

und folglich ist

$$
\frac{Q_{j}(\xi, \zeta)}{P(\xi, \zeta)}=\frac{Q_{j+}(\xi, \zeta)}{P_{+}(\xi, \zeta)}+\frac{Q_{j-}(\xi, \zeta)}{P_{-}(\xi, \zeta)}
$$

mit

$$
\frac{Q_{j}^{\prime}(\xi, \zeta)}{P^{\prime}(\xi, \zeta)}=\frac{Q_{j+}^{\prime}(\xi, \zeta)}{P_{+}^{\prime}(\xi, \zeta)}+\frac{Q_{j-}(\xi, \zeta)}{P_{-}(\xi, \zeta)}
$$

Daher gilt (vgl. 2.2)

$$
Q_{j+}^{\prime}(\xi, \zeta)=Q_{j+}(\xi, \zeta) / M(\xi, \zeta) \text {. }
$$

mit den Bezeichnungen

$$
\begin{aligned}
Q_{j}^{\prime}(\xi, \zeta)= & \sum_{\alpha \in \Lambda^{\prime}} \sum_{\beta \in Q_{\alpha}} q_{j \alpha \beta}^{+}(\xi)\left(\zeta-\zeta_{\alpha}(\xi)\right)^{\beta} P_{\alpha}^{\prime}(\xi, \zeta) \\
& +\sum_{\alpha \in \Lambda^{-}} \sum_{\beta \in Q_{\alpha}} q_{j \alpha \beta}^{-}(\xi)\left(\zeta-\zeta_{\alpha}(\xi)\right)^{\beta} P_{\alpha}^{\prime}(\xi, \zeta)
\end{aligned}
$$

und

$$
q_{j \alpha \beta}^{+}(\xi)=\left.\frac{1}{\beta !}\left(\frac{\partial^{\beta}}{\partial \zeta^{\beta}} \frac{Q_{j+}^{\prime}(\xi, \zeta)}{P_{+\alpha}^{\prime}(\xi, \zeta)}\right)\right|_{\zeta=\zeta_{\alpha}(\xi)}, \quad \alpha \in \Lambda^{\prime}, \quad \beta \in \varrho_{\alpha},
$$

$$
q_{j \alpha \beta}^{-}(\xi)=\left.\frac{1}{\beta !}\left(\frac{\partial^{\beta}}{\partial \zeta^{\beta}} \frac{Q_{j-}(\xi, \zeta)}{P_{-\alpha}(\xi, \zeta)}\right)\right|_{\zeta=\zeta_{\alpha}(\xi)}, \quad \alpha \in \Lambda^{-}, \beta \in \varrho_{\alpha} .
$$


Somit findet man infolge von (2.16)

$$
\begin{aligned}
\gamma_{0} Q_{j}^{\prime}\left(\xi, D_{t}\right)\left(\mathscr{F}_{x} v\right)(\xi, t)= & \sum_{\alpha \in \Lambda^{\prime}} \sum_{\beta \in \boldsymbol{e}_{\alpha}} q_{j \alpha \beta}^{+}(\xi) W_{\alpha, x_{\alpha}-\beta}(\xi) \\
& +\sum_{\alpha \in \Lambda^{-}} \sum_{\beta \in e_{\alpha}} q_{j \alpha \beta}^{-}(\xi) W_{\alpha, x_{\alpha}-\beta}(\xi) .
\end{aligned}
$$

Es gilt (man siehe 4.4)

Lemma 2.6. Die Funktionen $q_{j \alpha \beta}^{+}$und $q_{j \alpha \beta}^{-}$sind $q^{\prime}$-homogen (in $\xi$ ) mit

und

$$
\operatorname{deg}_{q^{\prime}} q_{j \alpha \beta}^{+}=\mu_{j}-\mu+\left(x_{\alpha}-\beta\right) q_{n}, \quad \alpha \in \Lambda^{\prime}, \quad \beta \in \varrho_{\alpha},
$$

$$
\operatorname{deg}_{q^{\prime}} q_{j \alpha \beta}^{-}=\mu_{j}-\mu+\left(\varkappa_{\alpha}-\beta\right) q_{n}, \quad \alpha \in \Lambda^{-}, \beta \in \varrho_{\alpha} .
$$

Wendet man jetzt die Lemmata 2.3, 2.4 und 2.6 an und benutzt die Stetigkeit von $q_{j \alpha \beta}^{-}$, so ergibt sich aus (2.19)

$$
\begin{gathered}
\langle\xi\rangle^{\mu-\mu_{j}-q_{n} / 2}\left|\sum_{\alpha \in A^{\prime}} \sum_{\beta \in \Theta_{\alpha}} q_{j \alpha \beta}^{+}(\xi) W_{\alpha, x_{\alpha}-\beta}(\xi)\right| \leqq\langle\xi\rangle^{\mu-\mu_{j}-q_{n} / 2}\left|\gamma_{0} Q_{j}^{\prime}\left(\xi, D_{t}\right)\left(\mathscr{F}_{x} v\right)(\xi, t)\right| \\
+C\left(\int_{-\infty}^{\infty}|f|^{2} d t\right)^{1 / 2} \sum_{\alpha \in \Lambda^{-}} \sum_{\beta \in \ell_{\alpha}}\langle\xi\rangle^{\left(x_{\alpha}-\beta-1 / 2\right) q_{n}}\left(\frac{1}{\left|\operatorname{Im} \zeta_{\alpha}(\xi)\right|^{2\left(x_{\alpha}-\beta\right)-1}}\right)^{1 / 2} .
\end{gathered}
$$

Damit haben wir wegen (2.14)

$$
\begin{gathered}
\langle\xi\rangle^{\mu-\mu_{j}-q_{n} / 2}\left|\sum_{\alpha \in \Lambda^{\prime}} \sum_{\beta \in e_{\alpha}} q_{j \alpha \beta}^{+}(\xi) W_{\alpha, x_{\alpha}-\beta}(\xi)\right| \\
\leqq\langle\xi\rangle^{\mu-\mu_{j}-q_{n} / 2}\left|\gamma_{0} Q_{j}^{\prime}\left(\xi, D_{t}\right)\left(\mathscr{F}_{x} v\right)(\xi, t)\right|+C\left(\int_{0}^{\infty}\left|P^{\prime}\left(\xi, D_{t}\right)\left(\mathscr{F}_{x} v\right)(\xi, t)\right|^{2} d t\right)^{1 / 2} \cdot
\end{gathered}
$$

2.7. Es sei $\xi \in \boldsymbol{R}^{n-1} \backslash\{0\}$. Wir behaupten, daß ein Vektor $\omega=\left(\omega_{\alpha \beta}\right)_{\alpha \in \Lambda^{\prime}, \beta \in \boldsymbol{Q}_{\alpha}}$ aus $C^{x}$ den Beziehungen

$$
\sum_{\alpha \in \Lambda^{\prime}} \sum_{\beta \in Q_{\alpha}} q_{j \alpha \beta}^{+}(\xi) \omega_{\alpha \beta}=0, \quad j=1, \ldots, \varkappa,
$$

nur dann genügen kann, wenn $\omega=0$ ist. Sonst hätte man nämlich

$$
\sum_{j=1}^{\varkappa} c_{j} q_{j \alpha \beta}^{+}(\xi)=0, \quad \alpha \in \Lambda^{\prime}, \quad \beta \in \varrho_{\alpha},
$$

mit gewissen Zahlen $c_{j} \in C, j=1, \ldots, \varkappa$. Dann wäre jedoch gemäß (2.16)

$$
\begin{aligned}
\sum_{j=1}^{x} c_{j} Q_{j}^{\prime}(\xi, \zeta)= & \sum_{j=1}^{x} c_{j} \sum_{\alpha \in \Lambda^{\prime}} \sum_{\beta \in Q_{\alpha}} q_{j \alpha \beta}^{+}(\xi)\left(\zeta-\zeta_{\alpha}(\xi)\right)^{\beta} P_{\alpha}^{\prime}(\xi, \zeta) \\
& +\sum_{j=1}^{x} c_{j} \sum_{\alpha \in \Lambda^{-}} \sum_{\beta \in \varrho_{\alpha}} q_{j \alpha \beta}^{-}(\xi)\left(\zeta-\zeta_{\alpha}(\xi)\right)^{\beta} P_{\alpha}^{\prime}(\xi, \zeta) \\
= & \left(\sum_{\alpha \in \Lambda^{-}} \sum_{\beta \in \varrho_{\alpha}}\left(\zeta-\zeta_{\alpha}(\xi)\right)^{\beta} P_{-\alpha}(\xi, \zeta) \sum_{j=1}^{x} c_{j} q_{j \alpha \beta}^{-}(\xi)\right) P_{+}^{\prime}(\xi, \zeta)
\end{aligned}
$$

im Widerspruch zu Bedingung (III). 
Für alle $\xi \in \boldsymbol{R}^{n-1} \backslash\{0\}$ gilt somit

$$
\sum_{j=1}^{x}\langle\xi\rangle^{2\left(\mu-\mu_{j}\right)}\left|\sum_{\alpha \in \Lambda^{\prime}} \sum_{\beta \in e_{\alpha}} q_{j \alpha \beta}^{+}(\xi) \omega_{\alpha \beta}\right|^{2}>0, \quad \omega=\left(\omega_{\alpha \beta}\right) \in C^{\alpha} \backslash\{0\} .
$$

Es sei nun $\Omega=\left(\Omega_{\alpha \beta}\right)_{\alpha \in \Lambda^{\prime}, \beta \in Q_{\alpha}} \in C^{\varkappa} \backslash\{0\}$. Setzen wir

so ist nach (2.21)

$$
\omega_{\alpha \beta}=\omega_{\alpha \beta}(\xi)=\langle\xi\rangle^{\left(\beta-x_{\alpha}\right) q_{n}} \Omega_{\alpha \beta},
$$

$$
\sum_{j=1}^{\varkappa}\langle\xi\rangle^{2\left(\mu-\mu_{j}\right)} \mid \sum_{\alpha \in \Lambda^{\prime}} \sum_{\beta \in Q_{\alpha}} q_{j \alpha \beta}^{+}(\xi)\langle\xi\rangle^{\left.\left(\beta-x_{\alpha}\right) q_{n} \Omega_{\alpha \beta}\right|^{2}>0 .}
$$

Der Ausdruck auf der linken Seite von (2.22) ist quadratisch in bezug auf die $\Omega_{\alpha \beta}$ derart, daß die Koeffizienten $q^{\prime}$-homogen in $\xi$ vom Grad 0 sind, was sich aus Lemma 2.6 folgern läßt. Demzufolge existiert aufgrund der Stetigkeit von $q_{j \alpha \beta}^{+}$ein solches $C_{5}>0$, daß die Ungleichung

$$
\sum_{j=1}^{\chi}\langle\xi\rangle^{2\left(\mu-\mu_{j}\right)}\left|\sum_{\alpha \in A^{\prime}} \sum_{\beta \in \varrho_{\alpha}} q_{j \alpha \beta}^{+}(\xi)\langle\xi\rangle^{\left(\beta-x_{\alpha}\right) q_{n}} \Omega_{\alpha \beta}\right|^{2} \geqq C_{5}
$$

für alle $\xi \in \boldsymbol{R}^{n-1},\langle\xi\rangle=1$, und $\Omega=\left(\Omega_{\alpha \beta}\right) \in C^{x},|\Omega|=1$, besteht, und somit gilt

$$
\sum_{j=1}^{\chi}\langle\xi\rangle^{2\left(\mu-\mu_{j}\right)}\left|\sum_{\alpha \in \Lambda^{\prime}} \sum_{\beta \in \varrho_{\alpha}} q_{j \alpha \beta}^{+}(\xi)\langle\xi\rangle^{\left(\beta-x_{\alpha}\right) q_{n}} \Omega_{\alpha \beta}\right|^{2} \geqq C_{5}|\Omega|^{2}=C_{5} \sum_{\alpha \in \Lambda^{\prime}} \sum_{\beta \in \varrho_{\alpha}}\left|\Omega_{\alpha \beta}\right|^{2}
$$

für alle $\xi \in \boldsymbol{R}^{n-1} \backslash\{0\}$ und $\Omega=\left(\Omega_{\alpha \beta}\right) \in \boldsymbol{C}^{x}$. Wählt man

so findet man

$$
\Omega_{\alpha \beta}=\Omega_{\alpha \beta}(\xi)=\langle\xi\rangle^{\left(x_{\alpha}-\beta-1 / 2\right) q_{n} W_{\alpha, x_{\alpha}-\beta}(\xi),}
$$

$$
\begin{aligned}
& \sum_{\alpha \in \Lambda^{\prime}} \sum_{\beta \in Q_{\alpha}}\left|\langle\xi\rangle^{\left(\alpha_{\alpha}-\beta-1 / 2\right) q_{n}} W_{\alpha, \alpha_{\alpha}-\beta}(\xi)\right|^{2} \\
& \quad \leqq C \sum_{j=1}^{\chi}\langle\xi\rangle^{2\left(\mu-\mu_{j}-q_{n} / 2\right)}\left|\sum_{\alpha \in \Lambda^{\prime}} \sum_{\beta \in Q_{\alpha}} q_{j \alpha \beta}^{+}(\xi) W_{\alpha, x_{\alpha}-\beta}(\xi)\right|^{2} .
\end{aligned}
$$

2.8. Die Beziehungen (2.15), (2.20) und (2.23) zusammen liefern

$$
\begin{aligned}
\int_{0}^{\infty}\left|R^{\prime}\left(\xi, D_{t}\right)\left(\mathscr{F}_{x} v\right)(\xi, t)\right|^{2} d t \leqq & C\left(\int_{0}^{\infty}\left|P^{\prime}\left(\xi, D_{t}\right)\left(\mathscr{F}_{x} v\right)(\xi, t)\right|^{2} d t\right. \\
& \left.+\sum_{j=1}^{\chi}\langle\xi\rangle^{2\left(\mu-\mu_{j}-q_{n} / 2\right)}\left|\gamma_{0} Q_{j}^{\prime}\left(\xi, D_{t}\right)\left(\mathscr{F}_{x} v\right)(\xi, t)\right|^{2}\right) .
\end{aligned}
$$

Falls wir hier über $\boldsymbol{R}_{\xi}^{n-1}$ integrieren, erhalten wir nach Anwendung der Parsevalschen Gleichung Abschätzung (2.1), womit Satz 2.1 bewiesen ist. 


\section{Die Notwendigkeit der Bedingungen}

Ein direkter Beweis der Notwendigkeit der Bedingungen (I) und (II) für das Erfülltsein von Abschätzung (1.1) läßt sich durch geringe Modifizierung von den in [5] benutzten Methoden führen. Deshalb werden wir nur die wesentlichen Schritte geben.

3.1. Es sei $\xi \in \boldsymbol{R}^{n-1} \backslash\{0\}$. Falls man als $u$ in (1.1) die durch

$$
u(x, t)=\left(\frac{1}{h}\right)^{(n-1) / 2} g\left(\frac{x}{h}\right) e^{i\langle x, \xi\rangle} v(t)
$$

erklärte Funktion nimmt, wobei $g \in C_{0}^{\infty}\left(R^{n-1}\right), v \in C_{0}^{\infty}\left[\bar{R}_{+}\right]$und $h>0$ ist, und $h \rightarrow \infty$ gehen läßt, so findet man

$$
\int_{0}^{\infty}\left|R\left(\xi, D_{t}\right) v\right|^{2} d t \leqq C\left(\int_{0}^{\infty}\left|P\left(\xi, D_{t}\right) v\right|^{2} d t+\sum_{j=1}^{\varkappa}\langle\xi\rangle^{2\left(\mu-\mu_{j}-q_{n} / 2\right)}\left|\gamma_{0} Q_{j}\left(\xi, D_{t}\right) v\right|^{2}\right)
$$

für alle $v \in C_{0}^{\infty}\left[\overline{\boldsymbol{R}}_{+}\right]$. Es genügt also den Fall $n=1$, d. h. die Abschätzung

$$
\int_{0}^{\infty}\left|R\left(D_{t}\right) v\right|^{2} d t \leqq C\left(\int_{0}^{\infty}\left|P\left(D_{t}\right) v\right|^{2} d t+\sum_{j=1}^{x}\left|\gamma_{0} Q_{j}\left(D_{t}\right) v\right|^{2}\right), \quad v \in C_{0}^{\infty}\left[\overline{\boldsymbol{R}}_{+}\right],
$$

$\mathrm{zu}$ betrachten.

3.2. Man kann voraussetzen, daß mit einem $\lambda^{\prime \prime}, 0 \leqq \lambda^{\prime \prime} \leqq \lambda^{\prime}$, gilt: $\zeta_{\alpha}$ ist eine Nullstelle des Polynoms $M(\zeta)$ mit der Ordnung $l_{\alpha}$ genau für $\lambda^{\prime \prime}<\alpha \leqq \lambda^{+}$. Wir setzen $\Lambda^{\prime \prime}=\left\{1, \ldots, \lambda^{\prime \prime}\right\}$ für $\lambda^{\prime \prime}>0$ und $\Lambda^{\prime \prime}=\emptyset$ für $\lambda^{\prime \prime}=0$ und haben dann

Falls man weiter

$$
l_{\alpha}= \begin{cases}k_{\alpha}-x_{\alpha} & \text { für } \alpha \in \Lambda^{\prime} \backslash \Lambda^{\prime \prime} \\ k_{\alpha} & \text { für } \alpha \in \Lambda^{+} \backslash \Lambda^{\prime}\end{cases}
$$

und

$$
s_{\alpha}=\left\{0, \ldots, l_{\alpha}-1\right\} \quad \text { für } \alpha \in \Lambda^{*}=\Lambda^{+} \backslash \Lambda^{\prime \prime}
$$

$$
\sigma_{\alpha}= \begin{cases}\varrho_{\alpha} & \text { für } \alpha \in \Lambda^{\prime \prime} \\ \left\{l_{\alpha}, \ldots, k_{\alpha}-1\right\} & \text { für } \alpha \in \Lambda^{\prime} \backslash \Lambda^{\prime \prime}\end{cases}
$$

setzt, so läßt die allgemeine Lösung der Gleichung $P_{+}\left(D_{t}\right) z=0$ sich in der Form

$$
z(t)=x(t)+y(t)=\sum_{\alpha \in \Lambda^{\prime}} \sum_{\beta \in \sigma_{\alpha}} x_{\alpha \beta} z_{\alpha \beta}(t)+\sum_{\alpha \in \Lambda^{*}} \sum_{\beta \in s_{\alpha}} y_{\alpha \beta} z_{\alpha \beta}(t), \quad x_{\alpha \beta}, y_{\alpha \beta} \in C,
$$

darstellen, wobei

ist. Nun gelten die Beziehungen

$$
z_{\alpha \beta}(t)=(i t)^{\beta} e^{i \zeta_{\alpha} t}
$$

$$
R\left(D_{t}\right) x=0 \quad \text { genau für } x_{\alpha \beta}=0, \alpha \in \Lambda^{\prime}, \beta \in \sigma_{\alpha},
$$


und

$$
R\left(D_{t}\right) y=0 \quad \text { für alle } y_{\alpha \beta}, \alpha \in \Lambda^{*}, \beta \in s_{\alpha} .
$$

Noch bemerken wir, daß Abschätzung (3.1) auch für $z$ besteht (vgl. [1], S. 682).

3.3. Wir zeigen zuerst, daß Bedingung (I) erfüllt ist. Aus (3.1) und (3.2) ergibt sich, daß

$$
\gamma_{0} Q_{j}\left(D_{t}\right) x=0, \quad j=1, \ldots, \varkappa,
$$

dann und nur dann gilt, wenn $x_{\alpha \beta}=0$ für alle $\alpha \in \Lambda^{\prime}, \beta \in \sigma_{\alpha}$ ist. Die Gleichungen (3.4) können mit Hilfe der Leibnizschen Formel (vgl. [3], S. 10) als Gleichungssystem

$$
\sum_{\alpha \in \Lambda^{\prime}} \sum_{\beta \in \sigma_{\alpha}} Q_{j}^{(\beta)}\left(\zeta_{\alpha}\right) x_{\alpha \beta}=0, \quad j=1, \ldots, \varkappa,
$$

in den Veränderlichen $x_{\alpha \beta}$ geschrieben werden, wobei $Q_{j}^{(\beta)}=\left(i D_{\zeta}\right)^{\beta} Q_{j}$ ist. Nach obigem ist die Koeffizientenmatrix dieses Systems nichtsingulär.

Somit kann man $x(t)$ in der allgemeinen Lösung $z(t)=x(t)+y(t)$ stets so auswählen, daß die Gleichungen

$$
\gamma_{0} Q_{j}\left(D_{t}\right) z=0, \quad j=1, \ldots, \varkappa,
$$

erfüllt sind; diese Gleichungen sind nämlich dem System

$$
\sum_{\alpha \in \Lambda^{\prime}} \sum_{\beta \in \sigma_{\alpha}} Q_{j}^{(\beta)}\left(\zeta_{\alpha}\right) x_{\alpha \beta}=-\sum_{\alpha \in \Lambda^{*}} \sum_{\beta \in s_{\alpha}} Q_{j}^{(\beta)}\left(\zeta_{\alpha}\right) y_{\alpha \beta}, \quad j=1, \ldots, \chi,
$$

äquivalent. Aus (3.1) folgt dann wegen (3.2) und (3.3), daß $x_{\alpha \beta}=0$ für alle $\alpha \in \Lambda^{\prime}$, $\beta \in \sigma_{\alpha}$ ist, und somit hat man gemäß (3.5)

$$
\sum_{\alpha \in \Lambda^{*}} \sum_{\beta \in \sigma_{\alpha}} Q_{j}^{(\beta)}\left(\zeta_{\alpha}\right) y_{\alpha \beta}=0, \quad j=1, \ldots, \varkappa .
$$

Aber dies kann der Fall nur dann sein, wenn für alle $j=1, \ldots, \varkappa$

$$
Q_{j}^{(\beta)}\left(\zeta_{\alpha}\right)=0, \quad \alpha \in \Lambda^{*}, \beta \in s_{\alpha},
$$

ist. Also gilt $Q_{j}(\zeta) \equiv 0 \bmod M(\zeta), j=1, \ldots, \varkappa$.

3.4. Wir zeigen noch, daß auch Bedingung (II) erfüllt ist. Zuerst bemerken wir, daß Abschätzung (3.1) für alle $v \in C_{0}^{\infty}\left[\overline{\boldsymbol{R}}_{+}\right]$dann und nur dann gilt, wenn die Ungleichung

$$
\int_{0}^{\infty}\left|R^{\prime}\left(D_{t}\right) w\right|^{2} d t \leqq C\left(\int_{0}^{\infty}\left|P^{\prime}\left(D_{t}\right) w\right|^{2} d t+\sum_{j=1}^{\varkappa}\left|\gamma_{0} Q_{j}^{\prime}\left(D_{t}\right) w\right|^{2}\right)
$$

für alle $w \in C_{0}^{\infty}\left[\overline{\boldsymbol{R}}_{+}\right]$besteht; jedes $w \in C_{0}^{\infty}\left[\overline{\boldsymbol{R}}_{+}\right]$läßt sich nämlich in der Form $w=M\left(D_{t}\right) v$ mit $v \in C_{0}^{\infty}\left[\overline{\boldsymbol{R}}_{+}\right]$darstellen (vgl. [1], S. 684).

Die allgemeine Lösung der Gleichung $P_{+}^{\prime}\left(D_{t}\right) z=0$ hat die Gestalt

$$
z(t)=\sum_{\alpha \in \Lambda^{\prime}} \sum_{\beta \in \varrho_{\alpha}} c_{\alpha \beta} z_{\alpha \beta}(t), \quad c_{\alpha \beta} \in C .
$$


Haben wir

oder

$$
\gamma_{0} Q_{j}^{\prime}\left(D_{t}\right) z=0, \quad j=1, \ldots, \varkappa,
$$

$$
\sum_{\alpha \in \Lambda^{\prime}} \sum_{\beta \in Q_{\alpha}} Q_{j}^{\prime(\beta)}\left(\zeta_{\alpha}\right) c_{\alpha \beta}=0, \quad j=1, \ldots, \chi,
$$

so folgt aus der (auch für $z$ geltenden) Ungleichung (3.6), da $\beta c_{\alpha \beta}=0$ für alle $\alpha \in \Lambda^{\prime}, \beta \in \varrho_{\alpha}$ sein muß. Gleichungssystem (3.7) besitzt also nur die triviale Lösung, und folglich sind die Polynome $Q_{j}^{\prime}(\zeta), j=1, \ldots, \varkappa$, linear unabhängig modulo $P_{+}^{\prime}(\zeta)$, woraus Bedingung (II) sich ergibt.

\section{Nachträgliche Beweise}

4.1. Beweis von Lemma 2.2. Es gilt

$$
\left(\mathscr{F}_{t} f\right)(\zeta)=\pi_{1} \int_{-\infty}^{\infty} e^{-i t \zeta} P^{\prime}\left(D_{t}\right) \tilde{\varphi}(t) d t=\pi_{1} \int_{0}^{\infty} e^{-i t \zeta}\left(D_{t}-\zeta_{\alpha}\right)^{\alpha_{\alpha}} P_{\alpha}^{\prime}\left(D_{t}\right) \varphi(t) d t .
$$

Für $z \in \boldsymbol{C}, g \in C_{0}^{\infty}\left[\overline{\boldsymbol{R}}_{+}\right]$und $s=1,2, \ldots$ findet man

und somit

$$
\begin{aligned}
\int_{0}^{\infty} e^{-i t \zeta}\left(D_{t}-z\right)^{s} g(t) d t= & (\zeta-z)^{s} \int_{0}^{\infty} e^{-i t \zeta} g(t) d t \\
& +i \sum_{k=1}^{s}(\zeta-z)^{k-1} \gamma_{0}\left(D_{t}-z\right)^{s-k} g(t)
\end{aligned}
$$

$$
\begin{aligned}
\left(\mathscr{F}_{t} f\right)(\zeta)= & \left(\zeta-\zeta_{\alpha}\right)^{\alpha_{\alpha}-\beta} \mathscr{F}_{t}\left(\left(D_{t}-\zeta_{\alpha}\right)^{\beta} P_{\alpha}^{\prime}\left(D_{t}\right) \tilde{\varphi}\right)(\zeta) \\
& +i \pi_{1} \sum_{k=1}^{\chi_{\alpha}-\beta}\left(\zeta-\zeta_{\alpha}\right)^{k-1} \gamma_{0}\left(D_{t}-\zeta_{\alpha}\right)^{\varkappa_{\alpha}-\beta-k}\left(D_{t}-\zeta_{\alpha}\right)^{\beta} P_{\alpha}^{\prime}\left(D_{t}\right) \varphi(t),
\end{aligned}
$$

woraus die Behauptung folgt.

4.2. Beweis von Lemma 2.3. In der Tat, man hat das Residuum

$$
\operatorname{Res}_{z=\zeta_{\alpha}} \frac{1}{\left(z-\zeta_{\alpha}\right)^{s}\left(z-\bar{\zeta}_{\alpha}\right)^{s}}=\frac{(-1)^{s-1}(2(s-1)) !}{((s-1) !)^{2}} \frac{1}{\left(\zeta_{\alpha}-\overline{\zeta_{\alpha}}\right)^{2 s-1}} .
$$

Beweis von Lemma 2.4. Nach Lemma 2.2 erhält man mit $\beta=x_{\alpha}-k$

$$
\frac{\left(\mathscr{F}_{t} f\right)(\zeta)}{\left(\zeta-\zeta_{\alpha}\right)^{k}}=\mathscr{F}_{t}\left(\left(D_{t}-\zeta_{\alpha}\right)^{\alpha_{\alpha}-k} P_{\alpha}^{\prime}\left(D_{t}\right) \tilde{\varphi}\right)(\zeta)+i \pi_{1} \sum_{s=1}^{k}\left(\zeta-\zeta_{\alpha}\right)^{s-k-1} W_{\alpha s} .
$$

Da die erste Funktion auf der rechten Seite sich analytisch fortsetzen läßt, folgt hieraus

$$
\int_{-\infty}^{\infty} \frac{\left(\mathscr{F}_{t} f\right)(\zeta)}{\left(\zeta-\zeta_{\alpha}\right)^{k}} d \zeta=i \pi_{1}\left(-2 \pi i W_{\alpha k}\right)=\pi_{1}^{-1} W_{\alpha k}
$$


4.3. Beweis von Lemma 2.5. Das folgende, leicht nachzuprüfende Lemma verallgemeinert eine bekannte Charakterisierung homogener Funktionen.

Lemma 4.1. Eine in $\boldsymbol{R}_{\eta}^{n} \backslash\{0\}$ differenzierbare Funktion $T$ (oder eine Distribution $T$ in $R_{\eta}^{n}$ ) ist genau dann $q$-homogen mit $\operatorname{deg}_{q} T=s$, wenn sie der verallgemeinerten Eulerschen Gleichung

genügt.

$$
\sum_{k=1}^{n} q_{k} \eta_{k} \frac{\partial T}{\partial \eta_{k}}=s T
$$

In diesem Falle, wenn $T$ außerdem $(\beta+1)$-mal stetig differenzierbar in $\boldsymbol{R}_{\eta}^{\boldsymbol{m}} \backslash\{0\}$ ist, ist $\left(\partial / \partial \eta_{n}\right)^{\beta} T$-homogen vom Grad $s-\beta q_{n}$.

Die in den durch (2.2) bzw. (2.3) erklärten Ausdrücken $r_{\alpha \beta}^{+}(\xi)$ bzw. $r_{\alpha \beta}^{-}(\xi)$ vorkommenden Polynome $P_{+\alpha}^{\prime}(\xi, \zeta), R_{+}^{\prime}(\xi, \zeta)$ und $P_{-\alpha}(\xi, \zeta), R_{-}(\xi, \zeta)$ sind $q$-homogen, und wenn man $\operatorname{deg}_{q} P_{+}$mit $\mu_{+}$bezeichnet, so gilt

$$
\begin{aligned}
& \operatorname{deg}_{q} P_{+\alpha}^{\prime}=\mu_{+}-m q_{n}-\varkappa_{\alpha} q_{n}, \\
& \operatorname{deg}_{q} R_{+}^{\prime}=\mu_{+}-m q_{n}, \\
& \operatorname{deg}_{q} P_{-\alpha}=\mu-\mu_{+}-\varkappa_{\alpha} q_{n}, \\
& \operatorname{deg}_{q} R_{-}=\mu-\mu_{+},
\end{aligned}
$$

wobei $m=\operatorname{ord}_{\zeta} M(\xi, \zeta)$ ist. Es sei jetzt $\xi \in R^{n-1} \backslash\{0\}$. Aufgrund von (2.13) und Lemma 4.1 erhalten wir dann für $t>0$

und analog

$$
r_{\alpha \beta}^{+}\left(t^{\left.q^{\prime} \xi\right)}=\left.\frac{1}{\beta !}\left(\frac{\partial^{\beta}}{\partial \zeta^{\beta}} \frac{R_{+}^{\prime}\left(t^{q^{\prime}} \xi, \zeta\right)}{P_{+\alpha}^{\prime}\left(t^{q^{\prime}} \xi, \zeta\right)}\right)\right|_{\zeta=\zeta_{\alpha}\left(t^{\left.q^{\prime} \xi\right)}\right.}=t^{\left(x_{\alpha}-\beta\right) q_{n}} r_{\alpha \beta}^{+}(\xi)\right.
$$

$$
r_{\alpha \beta}^{-}\left(t^{q^{\prime}} \xi\right)=t^{\left(x_{\alpha}-\beta\right) q_{n}} r_{\alpha \beta}^{-}(\xi)
$$

Die Behauptungen lassen sich hieraus wegen der Stetigkeit schließen.

4.4. Beweis von Lemma 2.6. In den Definitionen (2.17) bzw. (2.18) von $q\left({ }_{j \alpha \beta}^{+} \xi\right)$ bzw. $q_{j \alpha \beta}^{-}(\xi)$ sind auch die Polynome $Q_{j+}^{\prime}(\xi, \zeta)$ und $Q_{j-}(\xi, \zeta) \quad q$-homogen, und zwar mit

und

$$
\operatorname{deg}_{q} Q_{j+}^{\prime}=\mu_{j}-\mu+\mu_{+}-m q_{n}
$$

$$
\operatorname{deg}_{q} Q_{j-}=\mu_{j}-\mu_{+} \cdot
$$

Die Behauptungen können danach mit Hilfe von (2.13) und Lemma 4.1 gewonnen werden (vgl. 4.3). 


\section{Literatur}

[1] Gel'man, I. V., und V. G. MAZ'JA: Estimates on the boundary for differential operators with constant coefficients in a half-space. - Math. USSR-Izv. 8, 1974, 667-726.

[2] HöRmANDER, L.: On the theory of general partial differential operators. - Acta Math. 94, $1955,161-248$.

[3] HöRMANDER, L.: Linear partial differential operators. - [Third revised printing.] Die Grundlehren der mathematischen Wissenschaften 116. Springer-Verlag, Berlin-HeidelbergNew York, 1969.

[4] Matsuzawa, T.: On quasi-elliptic boundary problems. - Trans. Amer. Math. Soc. 133, 1968, $241-265$.

[5] MAZ'JA, V. G., und I. V. Gel'MAN: Estimates for differential operators with constant coefficients in a half-space. - Math. USSR-Sb. 25, 1975, 225-258.

[6] SChechter, M.: Integral inequalities for partial differential operators and functions satisfying general boundary conditions. - Comm. Pure Appl. Math. 12, 1959, 37-66.

[7] Schechter, M.: On the dominance of partial differential operators II. - Ann. Scuola Norm. Sup. Pisa Sci. Fis. Mat. (3) 18, 1964, 255-282.

Universität Jyväskylä

Mathematisches Institut

Sammonkatu 6

SF-40100 Jyväskylä 10

Finnland

Eingegangen am 3. Februar 1978 